\title{
FÉCONDITÉ ET PRATIQUES FUNÉRAIRES EN ÉGÉE A L'ÂGE DU BRONZE
}

\section{Robert Laffineur}

L'aspiration à la fécondité est assurément une des composantes principales des religions de l'Egée au cours de la période du Bronze, spécialement de la religion crétoise. Son origine remonte, comme ailleurs en Méditerranée, au fond des âges préhistoriques. Elle y apparaît comme une préoccupation spontanée, en réponse à une impérieuse nécessité vitale, mais elle ne s'y exprime encore que d'une manière bien élémentaire. Le monde minoen en donne une image incomparablement plus diversifiée, qui correspond, à n'en pas douter, à une conception plus mûrie et plus élaborée. On s'est maintes fois intéressé à la manifestation la plus évidente de cette aspiration, la place prépondérante, quasi exclusive, qu'occupe dans le culte crétois la notion de fertilité. ${ }^{1}$ Les documents archéologiques en avaient apporté, dès les premières fouilles, d'abondants témoignages. Mais l'intérêt s'est toujours concentré en priorité sur le domaine des vivants, alors qu'il y a des indices - moins nombreux, il est vrai, et plutôt continentaux que crétois - d'une relation entre les pratiques funéraires et la notion de fécondité. C'est à ces témoignages que je voudrais consacrer la présente communication. ${ }^{2}$ Elle ne prétend pas vider le sujet, mais, plus modestement, livrer les premiers résultats d'une réflexion et d'une recherche en cours. Elle s'attachera à quelques uns des aspects du problème, en attendant d'autres occasions de compléter, voire de préciser, les observations et les suggestions d'aujourd'hui.

On admettra aisément que le concept de fécondité s'assocįe assez naturellement avec le monde des morts. L'accession des défunts à une vie nouvelle dans l'au-delà, qui, à en juger par le principe et le contenu des mobiliers funéraires, devait faire partie des croyances fondamentales, ${ }^{3}$ suppose une sorte de seconde naissance et possède à ce titre des implications inévitables avec la fertilité et la fécondité.

On peut voir dans l'ensevelissement lui-même une garantie de régénération, à l'image de ce que montre le domaine végétal. Mais on peut être tenté de favoriser plus activement cette régénération par le choix de certaines offrandes funéraires. 
J'ai cru ainsi pouvoir établir, dans des études antérieures, que les ornements figurés de la parure du défunt manifestent l'existence, au début de l'epoque mycénienne, d'une iconographie symbolique. On y trouve "des images apotropaïques qui font office d'auxiliaires précieux dans le monde hostile de l'au-delà, chouette, aigle, masque complet ou réduit aux seuls yeux, et des figurations d'animaux, papillon, cigale, abeille, grenouille, triton, poulpe et peut-être cervidé, dont la faculté de métamorphose - voire de génération spontanée - la capacité de régénération ou les habitudes d'hibernation apparaissent comme autant de gages de survie après la mort ou d'accession à une vie nouvelle" (Laffineur 1985: 261). ${ }^{4}$ On peut de même, et la relation est plus directe et plus évidente, intégrer au mobilier funéraire des images ou des objets qui sont des symboles de fécondité. C'est ce que l'on observe, à nouveau au début de l'époque mycénienne, avec les petites figures en feuille d'or de femme portant les mains à la poitrine (Karo 1930-33: no. 36) de divinité nue aux oiseaux au geste identique (Ibid. no. $27-28)^{5}$, de divinité de type crétois au corsage ouvert et accompagnée de végétaux (Ibid., no. 75) ou avec un collier de perles en forme de grenade (Ibid., no. 77). ${ }^{6}$ La destination de ce dernier n'est peut-être pas exclusivement funéraire, comme semble l'indiquer la facture relativement soignée des perles. Une éventuelle utilisation primaire comme amulettes de fécondité ne modifie toutefois en rien les intentions exprimées par le motif: l'affectation des perles à la parure du défunt va probablement de pair avec une adaptation de la signification symbolique primitive du motif au monde de l'au-delà. La même remarque vaut sans doute pour les chatons de bague ornés d'une scène de culte de fertilité, ${ }^{7}$ conçus plus sûrement encore à l'origine comme ornements des vivants, mais choisis peut-être comme pièces de parure funéraire précisément en raison de la signification de leur décor. ${ }^{8}$ Elle vaut peut-être également pour la divinité aux végétaux, qui sert d'ornementation à une épingle en argent, ${ }^{9}$ et pour une des deux feuilles d'or à divinité aux oiseaux, que le système de fixation, constitué de clous à tête dorée (Ibid., no. 27), identifie comme un élément d'applique décorant peut être primitivement une pièce de mobilier. Quant aux autres feuilles à figure repoussée citées plus haut, elles paraissent avoir été, au contraire, réalisées spécialement pour les besoins funéraires et les petites perforations qui en marquent le contour permettent de penser à des ornements cousus au vêtement mortuaire. ${ }^{10}$

D'autres éléments du mobilier funéraire, etaient probablement chargés d'une signification en rapport avec la fécondité. C'est spécialement le cas, semble-t-il, des figurines en terre cuite, bien 
attestées durant toutes les phases du Bronze et dans toutes les régions de l'Egée. Un inventaire rapide permet déjà - à défaut d'une étude systématique qui ne peut être envisagée ici - d'observer une prédilection évidente pour des types liés plus ou moins directement au concept qui nous retient. L'exemple des variétés mycéniennes est spécialement significatif, avec les figurines féminines en phi et en kourotrophos, ainsi qu'avec les figurines animales, qui sont quasi exclusivement - est-ce un hasard? -des figurines de bovidé.11 Plus anciennement, les "idoles" cycladiques en marbre, qui ont été mises au jour principalement dans les sépultures, représentent une divinité qui n'est assurément pas étrangère au domaine de la fécondité (Pini 1968: 23). ${ }^{12}$

L'aspiration à la fécondité ne peut trouver cependant meilleure expression que dans l'incorporation d'oeufs d'autruche au mobilier funéraire. L'usage est attesté à l'époque mycénienne, avec les trouvailles du cercle A de Mycènes et de la tholos de Dendra. Les oeufs y sont toujours utilisés pour former un vase, en l'occurrence un rhyton, muni d'un col et d'un fond rapportés en faïence ou en or et éventuellement renforcé par un cerclage de bandes métalliques. ${ }^{13}$ Les équilvalents de ces pièces ne font pas défaut, en Crète (Dawkins 190304: 202; Platon 1971: 159; Touchais 1978: 762), à Mélos (Renfrew 1981a: 31, 1981b: 73) et à Théra (Foster 1979: 151-152 et pl. 53-54; Doumas 1983: pl. 42), mais ils proviennent la plupart du temps de contextes domestiques ou cultuels. ${ }^{14}$ Cela donne à penser que l'utilisation comme offrande funéraire doit être le résultat de ce que C. Renfrew appellerait volontiers une interpretatio Mycenaea (Renfrew 1981a: 32), 15 ou au moins d'une généralisation d'un usage apparemment secondaire en Crète. Les témoignages concernant l'utilisation de l'oeuf d'autruche en Méditerranée orientale ont été rassemblés tout récemment par A. Finet (1982: 69-77) et A. Caubet (1983: 193-198). La découverte de nombreux spécimens, entiers ou découpés en forme de vase, dans des sépultures, à Ur, Kish et Mari, au Luristan (Finet 1982: 72-74), en Syrie-Palestine et à Chypre (Caubet 1983: 194-195), ${ }^{16}$ met bien en évidence la connotation funéraire. Son origine - comme celle de la tradition de nos oeufs de Pâques - tient sans aucun doute au fait que l'oeuf est considéré naturellement comme un "symbole de la vie qu'il contient en germe" et, dans l'au-delà, comme un gage "de la vie future et de la résurrection qu'il promet au défunt" (Deonna 1922: 160). ${ }^{17} \mathrm{La}$ taille inhabituelle de l'oeuf d'autruche est propre, de surcroît, à frapper l'imagination et à passer pour une garantie supplémentaire de régénération et de fertilité, davantage encore que le nombre éléve d'oeufs que pond l'oiseau. ${ }^{18}$ 
Cette signification paraît confirmée par la présence de petites figures de dauphin sur un des oeufs de Mycènes (Karo 1930-33: no. 828). L'interprétation que $W$. Deonna a donnée de cette association (Deonna 1922: 157-166) est peut-être excessive. "L'image de l'oeuf cosmogonique" et "l'allusion au mythe de la naissance de la déesse, sortie de l'oeuf aquatique et poussée eu rivage par les dauphins" (Ibid., 166) répondent à des conceptions religieuses élaborées et organisées dont on hésite à admettre l'existence au milieu du deuxième millénaire. On préférera l'interprétation récente de $\mathrm{P}$. Somville, qui se situe en cette occurrence précise au niveau des réalités observables et des croyances intuitives plus générales et plus fondamentales qui en dérivent: "la mer, porteuse de vie et nourricière,...la dauphin, animal maternel par excellence..." dans lequel "l'homme projette un destin de promesses: la vie, la mort et peut-être une nouvelle naissance, après" (Somville 1.984: 4-7). Il n'y a là, après tout, que la juste affirmation d'une composante marine bien concevable en milieu égéen au niveau des croyances en une puissance fécondante universelle.

La valeur fécondante de l'élément liquide est également mise en évidence dans les libations funéraires. La pratique en est attestée en Grèce mycénienne. On a observé en particulier, au cours des fouilles, la présence fréquente, dans le dromos des tombes, parfois à proximité immédiate de l'entrée, de vases, principalement des kylix, qui avaient manifestement une fonction différente de celle de la vaisselle habituelle placée comme mobilier funéraire dans la tombe elle-même, et qui ont dû servir à des libations puis être jetés là après usage (Andronikos 1968: 93; Pini 1968: 70-71; Long 1974: 40). ${ }^{19}$ Mais au total les témoignages sont ici moins nombreux et moins diversifiés qu'aux époques ultérieures, protogéometrique et géometrique. On n'a pas, ou très peu, d'exemples des pratiques apparentées, plus ou moins régulièrement représentées au début du premier millénaire (Andronikos 1968: 93-97): vases placés sur la couverture de la sépulture, témoins d'un rite accompli au moment de la fermeture de la tombe; ${ }^{20}$ éléments tubulaires en terre cuite disposés verticalement et vases à fond perforé (Kübler 1954: 33-34), destinés à faciliter - en partie symboliquement sans doute - la pénétration du liquide dans le sol de la tombe. Les éléments tubulaires n'ont d'equivalents que dans les "tubes à serpents" minoens, mais il s'agit à nouveau de documents qui appartiennent au mobilier du culte, en l'occurrence du culte domestique, à Gournia et Koumasa par exemple, et leur fond n'est pas toujours percé. ${ }^{21}$ A moins qu'il ne faille faire le rapprochement - mais la distance chronologique est importante - avec les curieux "vases-pantalons", à une extrémité dédoublée, trouvés dans les tombes de la Mesara (Xanthoudides 1924: 
39, $94 \mathrm{pl}$. XXVIII et L), dont l'usage est incertain, mais dont la structure est de nature à suggérer une fonction comme instruments à libation. ${ }^{22}$ Quant aux vases à fond perforé, s'ils sont connus en assez grand nombre en Egée, ils proviennent eux aussi essentiellement de sites d'habitat ou de sanctuaires. ${ }^{23}$

On pourrait considérer cette apparente pauvreté en témoins de l'usage des libations funéraires à l'epoque mycénienne comme le résultat d'un concours de circonstances: les indices cités sont localisés généralement en dehors des tombes et on ne les a peut-être pas observés au cours de la fouille des sépultures. ${ }^{24}$ Et il est vrai que le type toujours bien défini des tombes de l'Egée, en particulier des tombes mycéniennes, n'invite pas effectivement à étendre les recherches aux abords de la fosse ou du dromos ou sur le pourtour de la chambre, dans des zones qui sont pourtant, les fouilles systématiques récentes l'on montré, ${ }^{25}$ susceptibles de livrer des vestiges importants. Mais en réalité, cette impression de pauvreté disparaît quand on veut bien chercher les témoignages ailleurs, c'est-àdire à l'intérieur même des tombes. On observe alors la présence dans le mobilier d'un autre type de rhytons, le vase en forme de tête animale, pour lequel on note, plus clairement encore que précédemment, car la documentation est ici plus abondante, la même opposition entre une utilisation cultuelle en Crète et une utilisation funéraire sur le continent mycénien.

On sait que le rhyton - l'étmymologie en témoigne - est, en raison de la présence d'un orifice de remplissage et d'un trou de vidange, le vase à libations par excellence dans le monde crétomycénien (Tuchelt 1962: 36-45; Koehl 1981: 179-188). Si l'origine des différentes variétés est incontestablement minoenne, ainsi que l'indique l'évolution générale des formes, aujourd'hui bien assurée (Koehl 1981; Petit 1984: 71-80), et si les exemplaires continentaux les plus anciens sont même probablement des objets crétois d'importation (Hood 1978: 163), il n'en reste pas moins que les contextes de trouvaille sont fondamentalement différents d'un domaine à l'autre et que la signification du rhyton devait être en conséquence différehte à Mycènes de ce qu'elle était en Crète. Il n'y a pas là contradiction: l'adoption d'une forme ou d'un motif étrangers - voire des deux simultanément quand il s'agit, comme ici, essentiellement de vases plastiques - n'implique pas nécessairement l'adoption de la signification et de l'utilisation particulières qui sont celles du modèle (Laffineur 1985: 264-265). C'est ce qu'a bien noté récemment R.B. Koehl, au terme d'un recensement de la documentation: "The evidence and interpretations presented here indicate that rhyta were used by the 
Minoans from Middle Minoan IIB to Late Minoan III in both domestic and cult activities. Rhyta first appear on the Greek Mainland in the Late Helladic I period. Most come from graves, although domestic contexts are also known. The presence on Crete of large groups of rhyta in repositories of cult implements is striking in contrast to the infrequency of rhyta in Mycenaean shrines. The presence of rhyta in Mycenaean graves and their absence in Minoan before Late Minoan III is another significant difference" (Koehl 1981: 187). ${ }^{26}$ En se reportant à l'usage général des rhytons, on verrait volontiers dans cette différence des contextes de trouvaille une préfiguration de la dichotomie classique des $\sigma \pi \circ \delta \delta \alpha$, libations destinées aux dieux, et des xo $i$, libations destinées aux défunts. ${ }^{27}$

C'est à ụne même distinction, sans pour autant la nommer, qu'aboutit, Ch. R. Long au terme de sa récente analyse des scènes du sarcophage peint d'Aghia Triada, encore qu'en l'occurrence les deux variétés soient intégrées au rituel funéraire. Le type de la scène de libation d'un des longs côtés du monument résulterait de l'association de deux rites distincts: une pratique de tradition minoenne, qui s'adresse à la divinité, ${ }^{28}$ et l'usage mycénien du "toast d'adieu" au défunt, qui implique sans doute un nombre plus élévé de participants que le premier rite et exige donc une quantité de liquide aussi importante que celle que l'on verse dans le cratère de l'extrémité gauche de la scène (Long 1974: 40, 73). ${ }^{29}$

Il semble que la destination des rhytons en tête animale déposés dans les tombes ne puisse correspondre à aucune de ces deux pratiques. La forme spécifique du récipient, bien différente de celle des cruches ou des coupes à boire, et surtout son caractère figuré inviteraient plutôt à y voir un ustensile utilisé pour une libation versée sur le sol même de la tombe, au moment de l'inhumation, et dont le défunt est la bénéficiaire naturel. L'écoulement d'un liquide semble de nature à évoquer et à susciter l'action d'une force régénératrice et l'efficacité du processus se trouve assurément renforcée quand le récipient prend la forme d'un animal, le plus fréquemment réduit à la seule tête, ${ }^{30}$ et peut-être davantage encore quand il s'agit de l'image du taureau, dont la puissance fécondante peut aisément passer pour une garantie supplémentaire de survie ou de nouvelle naissance. Les documents archéologiques incitent même à envisager la possibilité d'une libation de sang, le liquide de vie par excellence, qui conférerait à la terre et au défunt qui y est déposé sa propre substance vitale.

Le sarcophage d'Aghia Triada apporte ici à nouveau un témoignage de première importance. On y voit une représentation 
unique d'un sacrifice de taureau, dans lequel le sang de la victime est recueilli dans un récipient tronconique, équivalent de läuviovclassique. L'anse unique de celui-ci montre, on l'a bien noté, qu'il s'agit d'une forme différente de celle des seaux de la scène de libation du long côté opposé (Long 1974: 36, 62-63). ${ }^{31}$ L'observation a amené Ch. Long à y renonnaître non pas un vase complet posé sur le sol mais la partie supérieure d'un rhyton-cornet à demi enfoncé dans le sol (Ibid., 63), ${ }^{32}$ à travers lequel le sang peut pénétrer dans la terre et y accomplir son action vivifiante. ${ }^{33}$ Le principe des cavités à libations est bien attesté en Egée et Ch. Long en a rassemblé les exemples (Ibid., 63, n. 37). On y associera, d'un point de vue plus général, le témoignage des bassins des salles à pilier minoennes (Gesell 1985) et celui des nombreuses "salles lustrales", sans doute en partie au moins à destination cultuelle, dont le sol était enfoncé par rapport à celui des quartiers voisins. ${ }^{34}$ Quant à la représentation de l'enfoncement d'un élément dans le sol, on en a un autre exemple sur le sarcophage même avec la figure du défunt (Long 1974: 46) et un équivalent exact sur un vase-anneau de Mycènes (Ibid., 63, fig. 92). Le document appartient à une série bien connue, dont il sera question encore dans la suite. On observera seulement pour l'instant, à la suite de Ch. Long, que l'anneau supporte un vase à demi enfoncé qui est incontestablement un rhytoncornet, muni d'un petit bucrane en ronde bosse, allusion probable au sacrifice animal. On ajoutera que l'anneau dans lequel le rhyton s'enfonce représente sans doute le sol, puisqu'il est parsemé de fleurettes et sillonné par un serpent plastique. La libation est donc présentée ici aussi comme devant pénétrer profondément en terre. On notera encore que cette identification précise de l'áuviovdu sarcophage implique un rejet de l'hypothèse défendue par R. Paribeni (1908: 36, 57) et récemment par J. Sakellarakis $(1970: 185,193)$, selon laquelle le cratère de la face opposée était destiné à recevoir le sang du sacrifice. Si le sang de la bête immolée s'écoule au fur et à mesure dans le sol à travers un rhyton, on ne peut guère imaginer qu'il soit possible de le recueillir pour le vider dans un second récipient, même si celui-ci ne paraît pas d'une capacité excessive pour contenir le sang d'une seule victime (Long 1974: 69 n. 25).

Une seconde catégorie de témoignages concerne l'éventuelle pratique de sacrifices d'animaux daris le cadre des rites d'inhumation. Les ossements d'animaux ont été trouvés en grand nombre dans les tombes crétoises et mycéniennes, mais en l'absence de données précises sur les circonstances et les contextes de trouvaille on peut y voir indifféremment des restes de provisions de nourriture destinées au défunt, des reliefs du repas funèbre ou des vestiges de sacrifice (Pini 
1968: 27-28, 68-69; Sakellarakis 1970: 158-161;. Long 1974: 68). L'hésitation s'applique en particulier aux ossements de bovidés, pour lesquels le matériel disponible amène à constater une nouvelle fois l'opposition relevée précédemment: ils ont été trouvés en Crète essentiellement dans des contextes domestiques et cultuels et leur apparition dans les tombes de lîle, assez rare de surcroît, se situe à une époque récente et résulte sans doute d'une influence continentale. ${ }^{35}$ L'hypothèse du sacrifice vaut en tout cas sûrement pour la trouvaille de la tholos A d'Arkanes, de date MR IIIA, à laquelle J. Sakellarakis a consacré une étude extrêmement documentée. Il en ressort que le crâne de taureau déposé au milieu de l'entrée donnant accès à la chambre latérale est certainement - la fouille minutieuse ne laisse ici aucun doute - une partie isolée d'un animal sacrifié offerte au défunt à l'issue d'une immolation intervenue pendant le rituel d'inhumation (Sakellarakis 1970: 187-192). ${ }^{36}$ Le choix de la tête, partie essentielle s'il en est, n'est certes pas un hasard. Quant à l'usage de l'offrande de la tête pars pro toto, il est attesté ailleurs par les trouvailles archéologiques (Ibid., 188 n. 388), ${ }^{37}$ mais également par les têtes animales que l'on rencontre dans la glyptique et qui sont davantage, à coup sûr, que de simples motifs décoratifs de remplissage (Ibid., 173-174).

Dans ce contexte, l'usage des rhytons en forme de tête animale ou plutôt l'adaptation au rituel funéraire qu'en ont fait les Mycéniens - apparaît ainsi comme le résultat d'une association des deux principes qui viennent d'être examinés, celui de la libation de sang

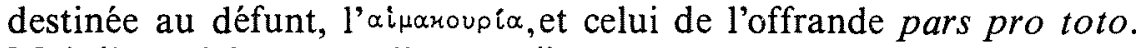
Mais il procède encore d'un troisième concept. La tête en terre cuite ou en métal précieux doit en effet être interprétée sans doute comme un substitut de la tête animale réelle et, à ce titre, comme un substitut du sacrifice réel..$^{38}$ Cette interprétation semble confirmée par le détail des cornes dorées dont sont pourvus les rhytons en tête de taureau en pierre ou en métal. G. St. Korres a montré récemment que l'application d'une couche de dorure sur les cornes des taureaux conduits au sacrifice est un usage bien attesté en Egée depuis le période créto-mycénienne jusqu'à l'epoque contemporaine (Korres 1973: 879-913 et 1977: 205221). La substitution pourrait tenir à des raisons d'économie, puisqu'aussi bien les exemples des tombes d'Arkanes et d'Aghia Triada se révèlent finalement assez isolés et concernent très probablement, ainsi que J. Sakellarakis l'a bien montré, des personnes de rang élevé (Sakellarakis 1970: 187-188). Mais la véritable raison est peut-être qu'une tête fabriquée dans un matériau non périssable est susceptible de remplir plus longtemps la fonction qu'on lui prête. L'ustensile avait, à n'en pas douter, un rôle effectif et ponctuel à jouer dans le rituel 
funéraire au moment de l'inhumation. ${ }^{39}$ Mais son incorporation au mobilier de la tombe assurait une présence que la résistance du matériau rend en quelque sorte indéfinie et permettait ainsi de prolonger éternellement, de manière virtuelle, le rituel de libation régénératrice dont bénéficie le défunt. On a là la manifestation d'une croyance quasi universelle, selon laquelle l'accomplissement des rites se continue grâce à la seule présence de l'ustensile adéquat, de la même manière qu'il se poursuit virtuellement par l'intermédiaire de la représentation figurée du rite lui-même (Cassimatis 1973: 122). On a là également une manifestation de la tendance à l'expression symbolique qui paraît bien caractériser l'art mycénien et conférer aux objets et aux images une valeur active. C'est précisément dans le contexte funéraire que cette conception trouve les applications les plus nombreuses. ${ }^{40} \mathrm{On}$ connaît déjà les éléments de parure funéraire mentionnés plus haut, dont l'iconographie est fréquemment en rapport avec la protection ou la régénération du défunt et qui apportent ainsi symboliquement une sorte de garantie pour l'au-delà. 41 On citera également l'usage des masques funéraires et autres plaques de revêtement corporel, dont la fonction était de dissimuler les effets de la décomposition du corps, ou au moins de sa partie considérée comme essentielle, et de conserver au défunt les apparences de l'existence (Laffineur 1985: 252). La plupart de ces documents sont réalisés en or, matériau inaltérable, aisément compris comme un gage de la pérennité des vertus magiques que l'on prête aux ornements funéraires. L'efficacité de ces derniers est encore accrue par le caractère volontiers emblématique de l'imagerie et par la répétition fréquente des motifs (Vermeule 1975: 16-18, 23, 25, 40-47).

La même signification d'ustensile de libation symbolique était probablement attachée aux rhytons-cornets trouvés dans des contextes funéraires (Pini 1968: 67) ${ }^{42}$ et on doit admettre ici aussi une adaptation d'une forme d'usage sans doute à l'origine essentiellement cultuelle (Koehl 1981: 184-186).

L'interprétation s'applique aussi aux vases-anneaux de type élaboré, mais ces récipients sont surtout typiques du domaine chypriote et leur utilisation se prolonge abondamment au début du premier millénaire, dans les sanctuaires et surtout dans les tombes. ${ }^{43} \mathrm{Ils}$ méritent de faire à eux seuls l'objet d'un examen détaillé qui dépasse largement le cadre de la présente contribution. En se limitant à des observations générales, on remarque toutefois que les éléments fixés sur l'anneau sont le plus souvent des petits vases qui communiquent avec le corps principal du récipient et permettaient donc d'assurer l'écoulement d'un liquide dans la partie annulaire. Mais on note aussi 
la présence d'autres éléments constitutifs en ronde bosse, qui sont manifestement en relation avec les rites examinés plus haut et, plus généralement, avec l'idée de fécondité: tête de taureau, munie habituellement de l'orifice de vidange de l'ensemble du vase, ${ }^{44}$ figure de grenouille, ${ }^{45}$ grenade, ${ }^{46}$ coquillage. ${ }^{47}$

On rangera enfin dans cette même catégorie des instruments de libation funéraire les coquilles de triton et leurs imitations en pierre, en terre cuite ou en faïence, dont les exemplaires ont été rassemblés récemment par $\mathrm{P}$. Darcque dans le cadre de la publication du triton en pierre MRIA de Malia (Baurain-Darcque 1983: 59-73). ${ }^{48}$ On a montré à cette occasion qu'une identification de ces documents comme des rhytons $^{49}$ devait être préférée à l'interprétation habituelle qui veut y voir, sans arguments déterminants, des trompettes à usage rituel (Ibid., 52-58). ${ }^{50} \mathrm{Si}$ le contexte de trouvaille de ces objets est souvent domestique ou cultuel, il y a cependant aussi de nombreux exemples de leur association au mobilier des tombes, au MR principalement. ${ }^{51} \mathrm{La}$ valeur d'instrument de libation funéraire, réelle puis symbolique, doit sans doute être admise ici aussi. Cela est d'autant plus vraisemblable que l'on prêtait dans l'antiquité au triton une faculté de génération spontanée et que son mode de vie se caractérise par une période d'hibernation (Laffineur 1985: 257-259). Ces deux particularités n'ont certes pas le même degré de réalité, mais elles sont de nature à passer pour des garanties de l'accession à une vie nouvelle dans l'au-delà. C'est le sens qu'il faut donner aux petites figures de triton en or de la tholos 3 de Peristeria (Marinatos 1967: 12, fig. 16). C'est probablement aussi la signification qu'il convient d'accorder aux tritons naturels et à leurs imitations. De même que pour les vases en forme d'animal ou de tête, l'écoulement du liquide de libation, qu'il soit effectif ou virtuel, était sans doute conçu ici comme le moyen symbolique de transférer la substance et les vertus régénératrices du mollusque à l'intérieur de la tombe. Un principe analogue a présidé à la conception des rhytons en forme de buste féminin aux seins perforés trouvés dans les nécropoles de Mochlos $^{52}$ et de Malia. ${ }^{53}$ Le lien est là plus direct encore avec la fécondité, mais le fonctionnement symbolique du récipient n'est pas différent.

Au terme de la présente étude, on perçoit mieux la relation qui s'est établie en milieu égéen entre les pratiques funéraires et la notion de fécondité. On a pu constater à plusieurs reprises le rôle décisif que paraissent avoir joué les Mycéniens dans l'enrichissement de cet aspect des conceptions funéraires, en particulier par l'adaptation qu'ils ont faite d'usages traditionnels empruntés au domaine cultuel. Mais il 
convient d'être prudent dans les conclusions que l'on peut tirer de cette observation. Le hasard des trouvailles est peut-être responsable, dans une mesure non négligeable, de la différence fondamentale que l'on a pu noter plus d'une fois entre les pratiques minoennes et mycéniennes. Les tombes crétoises du MM et du début MR ne sont connues qu'en nombre relativement restreint et une lacune correspondante concerne par ailleurs les sites d'habitat du début de l'époque mycénienne (Koehl 1981: 187 no. 40). Il n'est pas douteux non plus que les rites dont les traces nous sont parvenues sont les plus élaborés et sans doute ceux qui étaient accomplis en l'honneur de défunts de haut rang. Le bilan, dès lors, serait à coup sûr différent si l'on pouvait faire intervenir les données concernant la masse des inhumations des classes moyenne et inférieure (Sakellarakis 1970: 188; Long 1974: 50). Il faut enfin, au moment de conclure, se garder également d'oublier que les vestiges matériels des rites funéraires sont d'importance éminemment inégale et que l'essentiel de leurs manifestations nous échappe ainsi probablement en grande partie, d'autant qu'il s'agit de phénomènes de nature essentiellement spirituelle. Mais quoi qu'il en soit, une chose paraît certaine. Par leurs implications avec le concept de fécondité, les croyances et les rites qui accompagnent la mort sont étonnamment proches dans le monde égéen de ceux qui s'attachent à la naissance. Les deux moments primordiaux de la vie se trouvent ainsi réunis, comme les deux composantes de la dichotomie fondamentale de l'existence, ou, pour reprendre l'heureuse formule de P. Somville, comme une incarnation de "la même constante anthropologique de la dualité attrayante-fascinante de qui donne la vie et la reprend, incarne et désincarne, fait croître et se corrompre, alternativement" (Somville 1984: 4-5).

\section{Notes}

1 Voir notamment à ce propos Ch. Picard, Les réligions préhelléniques (Crète et Mycènes), Paris 1948 et M.P. Nilsson, The Minoan-Mycenaean Religion and its Survival in Greek Religion, 2e éd., Lund 1950, passim. La bibliographie à ce sujet a été rassemblée dans I.M. Ruud, Minoan Religion. A Bibliography, Oslo 1980 (voir l'index, p. 57, s.v. Mother Goddess).

2 Il sera question ici des pratiques liées à l'inhumation propremnet dite, non des manifestations possibles d'un culte du défunt accompli après la mise en terre, de manière répétée, éventuellement à intervalles réguliers. La différance a bien été précisée par G.E. Mylonas, qui a justement mis en garde contre toute confusion dans ce domaine: The Cult of the Dead in Helladic Times, Studies Presented to David Moore Robinson, I Saint Louis 1951, p. 64-65. 
3 Sur l'au-delà, voir Picard, op. cit., p. 161-175 et Nilsson, op, cit., p. 619-633. La pratique fréquente du "nettoyage" des sépultures pour faire place à des nouvelles inhumations implique peut-être seulement la croyance et une survie brève. Voir à ce propos Mylonas, op. cit., p. 92 et 98-99; id., Homeric and Mycenaean Burial Customs, $A J A, 52,1948$, p. 70-71; Cassimatis 1973: 121 ("...il n'est pas impossible qu'il existat [sic] la croyance en une survie très courte: le temps que prend le corps à se décomposer, ou le temps pour la vie "spirituelle" de passer dans un autre monde. Le mobilier servirait à faciliter ce passage, en assistant le défunt dans son attente et ses transactions avec les forces souterraines, après quoi, corps et offrandes perdant leur importance pouvaient être impunément violés) et 165 ("Mais la tombe n'est pas la demeure de la vie future. Elle sert à faciliter le passage d'un état à un autre et les offrandes ouvrent la voie tout en aidant le mort dans cette transformation et en sauvegardant sa personnalité. Sinon, chaque caveau resterait inviolé"). Voir aussi Long 1974: 18, 32, 46 (le sarcophage d'Aghia Triada utilisé au moins deux fois successivement). Contra, Pini 1968: 73.

4 Pour le symbolisme de protection, voir R. Laffineur, Le symbolisme funéraire de la chouette, $A C 50,1981$, p. $432-444$. On observe les indices de croyances analogues en rapport avec le monde végétal: certaines plantes sont naturellement considérées, de même que leur simple figuration, comme gages de fertilité, en particulier de la régénération annuelle de la vie végétale. Le fait,a bien été mis en évidence récemment par $\mathrm{P}$. Warren à propos de la scille de mer, mais les témoignages recueillis concernent exclusivement le domaine cultuel (Of Squills, Aux origines de l'hellénisme. La Crète et la Grèce. Hommage à Henri van Effenterre, Paris 1984, p.17-24). On peut s'étonner que les vertus de cette plante n'aient apparemment pas été mises à profit dans le contexte funéraire, étant donné la connotation d'immortalité qui s'y attache aujourd'hui encore (op. cit., p. 17).

5 Pour l'interprétation de l'oiseau, voir Nilsson, op. cit.,p. 330-340.

6 Pour le symbolisme de la grenade, voir RE, s.v. Malum Punicum, col. 939-940.

7 Voir les nombreux exemples recensés dans CMS, passim et E.T. Vermeule, Götterkult, Archaeologia Homerica, V, Göttingen 1974, p. 14-16.

8 L'iconographie mycénienne exprime également d'autres formes de symbolisme. Voir à ce propos R. Laffineur, Iconographie mycénienne et symbolisme guerrier, Art \& Fact. Revue des historiens d'art, archéologues et musicologues de l'Université de Liège, 2, 1983, p. 38-49.

9 Les détails de la description de Karo 1930-33: 54, no. 75 montrent que l'association des deux éléments n'est pas douteuse.

10 Sur l'usage du vêtement mortuaire, voir Andronikos 1968: 7, 9, 97.

11 Sur les terres cuites mycéniennes, voir l'étude fondamentale d'E. French, The Development of Mycenaean Terracotta Figurines, BSA, 66, 1971, p. 101-187 (spécialement p. 107-108 pour l'interprétation) et la mise au point récente de la même spécialiste, Mycenaean Figures and Figurines, their Typology and Function, Sanctuaries and Cults in the Aegean Bronze Age, Proceedings of the First International Symposium at the Swedish Institute in Athens (1980), Stockholm 1981, p.173-178. Voir aussi pour l'interprétation G.E. Mylonas, Mycenae and the Mycenaean Age, Princeton 1966, p. 114-116; Andronikos 1968: 98 et Long 1974: 47.

12 Une interprétation apparentée mais plus ébalorée a été proposée par J. Thimme: les "idoles" seraient les images d'une divinité astrale et constitueraient pour le défunt une garantie magique de renaissance (voir en dernier lieu dans Kunst und Kultur der Kykladeninseln im 3. Jahrtausend v. Chr., Karlsruhe 1976, p. 453-456).

13 Mycènes, tombe IV: Karo 1930-33: no. 552 (oeuf), 567 et 573 (embouchure et fond en faïence), avec traces d'un revêtement en feuille de bronze: Mycènes, tombe V: Karo 1930-33: no. 828 (oeuf avec dauphins en faïence appliqués) et 651 (fond en or), no. 832 (fragment d'oeuf) et 774 (embouchure en faïence); tholos de Dendra: A.W. Persson, The Royal Tombs at Dendra near Midea, Lund 1931, p. 37 et 54 et pl. III (bandes de bronze doré). Pour la reconstitution des exemplaires de Mycènes, voir Foster 1979: 130-134, fig. 87-88 et pl. 41-42. Un autre spécimen est mentionné dans A.W. Persson, New Tombs at Dendra, Lund 1942, p. 146. Les bandes en bronze de l'oeuf de Dendra correspondent sans doute à la dénomination ko-no-ni-pi / kononiphi, "avec des barres, des bandes", qui accompagne l'idéogramme *217 VAS sur la tablette linéaire B KN K 434 (Fr. Vandenabeele et J.P. Olivier, Les idéogrammes archéologiques du linéaire $B$, Paris 1979, p. 241-245).

14 Seul, l'exemplaire de Palaikastro (Dawkins 1903-04: 202) provient d'un contexte funéraire. 
15 La priorité chronologique des spécimens de Mycènes, que l'on pourrait envisager, inviterait à retenir le processus inverse d'une signification primitivement funéraire - et originaire de l'Orient, ainsi qu'on va le voir - adaptée ensuite en Crète et dans les îles au domaine cultuel. Mais si cette priorité semble confirmée par les trouvailles de Théra (Foster 1979: 151-152) et peut-être par celles de Phylacopi et de Zakro, les contextes respectivement MA et MMIII des rhytons trouvés à Palaikastro et à Kommos (Touchais 1978: 762) obligent à écarter cette hypothèse. On le fera d'autant plus facilement que les imitations en terre cuite de vases en oeuf d'autruche remontent en Crète jusqu'au MMII (Sir A. Evans, The Palace of Minos, I, Londres 1921, fig. 436, entre les p. 594-595 et II, Londres 1928, fig. 129; voir aussi G. Walberg, Kamares. A Study of the Character of Palatial Middle Minoan Pottery, Uppsala 1976, p. 42) et que les oeufs de Mycènes ne sont sans doute, avec leurs accessoires en faïence, que des produits crétois d'importation, ou du moins des productions inspirées de modèles crétois (Vermeule 1975: 1920 et Foster 1979: 134, 136-137).

${ }^{16}$ Les oeufs d'autruche sont également attestés dans les tombes puniques. Voir en dernier lieu M. Ponsich, Recherches archéologiques à Tanger et dans sa région, Paris 1970, p. 130-140 et P. Cintas, Manuel d'archéologie punique, II, Paris, 1976, p. 282-284.

17 Sur la signification funéraire de l'oeuf, voir aussi J. Wiesner, Grab und Jenseits, Berlin 1938, p. 218, et M.P. Nilsson, Das Ei im Totenkult der Alten, Opuscula selecta, I, Lund 1951, p. 3-20.

18 Aristote, H. Anim., IX, 15, 616b5; Aristote, Gen. Anim., III, 1, 749b17; Pline, H.N., X, 143. Voir à ce propos $R E$, s.v. Strauss, col. 341-342. Les autres raisons que l'on peut invoquer paraissent moins déterminantes: l'oeuf d'autruche déposé comme nourriture pour le défunt (Finet 1982: 72, n. 26) ou considéré comme symbole de résurrection parce qu'il éclôt de luimême quand il est enfoui dans le sable (Deonna 1922: 160, n. 9).

19 Les nombreux vases trouvés dans les pièces annexes des tombes circulaires de la Mesara lesquelles ne contenaient pas d'inhumations - témoignent sans doute des mêmes pratiques (Pini 1968: 29-30; K. Branigan, The Tombs of Mesara, Londres 1970, p. 98-103). Pour la Crète du Bronze récent, voir aussi Long 1974: 66.

20 Le mobilier de la tombe à fosse 36 de Zafer Papoura à Cnossos (la "chieftain's grave") est disposé pareillement sur la dalle de couverture (Pini 1968: 62 et fig. 81), mais il ne s'agit pas de vases spécialement destinés aux libations.

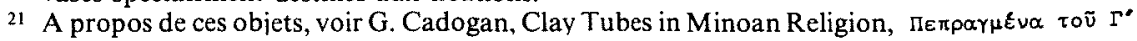

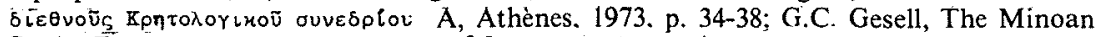
Snake Tube, $A J A, 77.1973$, p. $213-214$ et The Minoan Snake Tube: A Survey and Catalogue, $A J A, 80,1976$, p. 247-259.

22 Le rapprochement avec des cônes de Malia (Pini 1968: 26) n'éclaire pas la question, car ces objets n'ont qu'une extrémité perforée (l'extrémité étroite).

23 A. Maiuri, Jalisos, ASAtene, 6-7, 1923-1924, no. 7, p. 131 et fig. 51 (lalysos, tombe 19); J. -Cl. Poursat, Un sanctuaire du Minoen Moyen II à Mallia, $B C H, 90,1966$, no. 11, p. 537 et fig. 2829, p. 538; Koehl 1981: 181, fig. 2a-d; P. Warren, Minoan Crete and Ecstatic Religion, Sanctuaries and Cults..., p. 156 et Of Squills, Aux origines de l'hellénisme..., p. 20 et pl. VIII, 1-2 ("cup-rhyton"); W.W. Cummer et E. Schofield, Keos, III, Ayia Irini: House A, Mayence 1984, no. 999, pl. 30; B. Wells, Asine II, 4, The Protogeometric Period, 1, The Tombs, Stockholm 1976, fig. 32, p. 25 (date incertaine, peut-être HR).

24 Voir cependant l' "autel" situé au-dessus de la tombe IV du cercle de l'acropole de Mycènes (en dernier lieu L. Pelon, Tholoi, tumuli et cercles funéraires, Paris 1976, p. 146-147) et, en Crète, les quelques exemples de fosses à mobilier séparées des sépultures proprement dites (Pini 1968: 31).

25 Voir par exemple le mur de péribole circulaire autour de la grande tholos de Peristeria et le

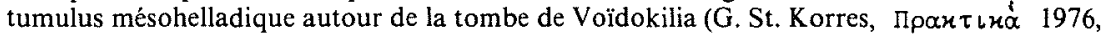
p. $481-485$ et 1977 , p. $242-295$ ).

26 Les rhytons en tête animale sont rassemblés dans Sakellarakis 1970: 191, n. 429. Les seuls exemplaires crétois trouvés dans une tombe sont les fragments de la "tombe des doubles haches" de Cnossos (Sir A. Evans, The Tomb of the Double Axes and Associated Group and Pillar Rooms and Ritual Vessels of the 'Little Palace' at Knossos, Londres 1914 [Archaeologia LXV], p. 72, fig. 50), un spécimen de Ligortyno (E. Pottier, Documents céramiques du Musée du Louvre, $B C H, 31,1907$, p. 117, no. 1 et pl. XXIII, en haut) et, peut-être, un rhyton inédit de 
Platanos mentionné par J.A. Sakellarakis. Le deuxième document est cependant daté du MRIIIA, c'est-à-dire de l'époque de l'occupation mycénienne en Crète.

27 Sur ces deux formes de libations, voir notamment J. Rudhardt, Notions fondamentales de la pensée religieuse et actes constitutifs du culte dans la Grèce classique, Genève 1958, p. 240-248.

28 Sans doute une divinité (ou des divinités) protectrice du défunt comme sur le modèle en terre cuite de la tombe de Kamilari (Long 1974: 45-46; pour le monument, voir D. Levi, La tomba a tholos di Kamilari presso a Festos, ASAtene, 39-40, 1961-1962, p. 123-139.

29) Cette pratique du "toast d'adieu" peut se restituer d'après les nombreuses trouvailles de fragments de coupes à boire découvertes dans le dromos des tombes (supra,p.82).

30 Outre les exemples de têtes dont il a été question plus haut (supra, n. 26), on mentionnera les rhytons en forme d'animal entier, en particulier ceux des tombes de la Mesara (Branigan, $o p$. cit., p. 81).

31 J.A. Sakellarakis note également cette différence, mais il estime qu'il s'agit pourtant d'un vase à deux anses et avoue l'impossibilité d'en trouver des équivalents (Sakellarakis 1970: 180).

32 On préférera cette hypothèse à celle d'un seau sans fond proposée par E.O. James (The Cult of the Mother-Goddess, Londres 1959, p. 131). On observera toutefois que la cavité où s'enfonçait le vase devait être assez profonde, faute de quoi le trou de vidange de l'extrémité inférieure du vase aurait été en contact avec le fond de la fosse, qui l'aurait obturé, rendant ainsi impossible l'écoulement du liquide.

${ }_{33}$ L'idée est bien exprimée par E.O. James (op. cit., p. 131): “...the life-giving blood of the victim being conveyed to them [the deceased] by means of ritual jars which carry the offering to Mother-earth, regarded as the ultimate source of rebirth". Voir aussi Wiesner, op. cit., p. 187188.

34 Voir en dernier lieu à leur propos J.W. Graham, Bathrooms and Lustral Chambers, Greece and the Eastern Mediterranean in Ancient History and Prehistory. Studies Presented to Fritz Schachermeyr, Berlin et New York, 1977, p. 110-125.

35 Voir la liste des trouvailles dans Sakellarakis 1970: 215-217. La présence d'ossements de bovidés n'est attestée qu'une seule fois dans un sanctuaire à l'époque mycénienne (Sakellarakis 1970: 164).

36 L'auteur admet que le sacrifice se faisait hors de la tombe, à l'air libre. Il note un seul cas de sacrifice accompli à l'intérieur de la sépulture, mais l'indice utilisé, la prétendue table de sacrifice en pierre du "cénotaphe" de Dendra (A. W. Persson, The Royal Tombs at Dendra near Midea, Lund 1931, pl. XXIX, à gauche), doit être aujourd'hui rejeté. Le document a été réinterprété récemment de manière convaincante par $\dot{A}$. Äkerström comme le support d'un sarcophage en bois (Mycenaean Problems, Op. Ath., 12, 1978, p. 69-73). Sur la valeur sacrificielle du taureau et de son sang, voir aussi M.C. Loulloupis, The Position of the Bull in the Prehistoric Religions of Crete and Cyprus, Acts of the International Archaeological Symposium "The Relations between Cyprus and Crete ca. 2000-500 B.C.", Nicosie 1979, p. 216-217.

37 Sur le principe de l'offrande partielle, voir aussi Pini 1968: 68.

38 Les figurines animales des sanctuaires et des tombes sont interprétées pareillement comme des substituts du sacrifice, de même que la représentation du rituel, sur le sarcophage d'Aghia Triada par exemple (Pini 1968: 28, 70).

39 Le liquide versé à cette occasion n’ètait probablement pas du sang, qui aurait nécessité un sacrifice, mais un liquide également de substitution, peut-être du vin (Long 1974: 68) ou simplement de l'eau.

40 Les indices de l'existence d'un symbolisme guerrier sont toutefois également observables (voir supra, n. 8).

41 Supra, p.80.

42 Les spécimens mycéniens sont rassemblés dans A. Furumark, The Mycenaean Pottery. Analysis and Classification, Stockholm 1942, p. 618 (type 199).

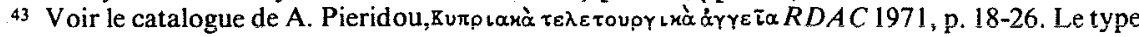
est attesté également à Mycènes (Furumark, op. cit., p. 618, type 197).

44 Pieridou, op. cit., pl. IX-XI; H.-G. Buchholz et V. Karageorghis, Altägäis und Altkypros, Tübingen 1971, no. 1271; E.T. Vermeule, Götterkult, Archaeologia Homerica, V, Göttingen 1974, pl. VIIb. Ce dernier document est un spécimen exceptionnel de l'Héraion de Samos, daté des environs de 600 et de fabrication probablement rhodienne (voir H. Walter et Kl. Vierneisel, 
Heraion von Samos. Die Funde der Kampagnen 1958 und 1959, $A M, 74,1959$, p. 29-30 et Beil. 67 et Kl. Vierneisel, Neue Tonfiguren aus dem Heraion von Samos, $A M, 76,1961$, p. 28-34 et 52-59 et Beil. 24-32). La même signification symbolique était peut-être attachée à un trépied en stéatite à tête de taureau de la collection Stathatos (D. Levi, Mobilier funéraire de Kharvati, Collection Hélène Stathatos, III, Strasbourg 1963, no. 8, p. 24-25 et 28).

45 Sur le vase-anneau de l'Héraion de Samos cité à la note précédente. Sur la signification symbolique de la grenouille, voir Laffineur 1985: 257.

46 Sur le même vase de Samos et dans Pieridou, op. cit., pl. X, 1 et XI, 1-2.

47 Sur le vase de Samos. Pour le signification du coquillage, voir ci-dessous.

48 Pour les trouvailles de coquillages en faïence, voir aussi Foster, op. cit., p. 83-85 et 137-140.

49 L'absence de perforation à l'extrémité pointue de certains spécimens (P. Warren, Minoan Stone Vases, Cambridge 1969, p. 91) n'est pas suffisante pour écarter l'interprétation comme vases à libations. Comme le font observer $\mathrm{Cl}$. Baurain et $\mathrm{P}$. Darcque, les rhytons ne sont pas les seuls vases à libation et il faut, dans le cas des tritons sans orifice de vidange "supposer que le liquide offert était introduit, puis reversé par la même ouverture"(Baurain-Darcque 1983: 57).

50 Pour une signification possible - mais peu vraisemblable - de cet usage dans le contexte funéraire, voir Long 1974: 13-14.

s1 Aux spécimens repris dans le catalogue de P. Darcque (Baurain-Darcque 1983: no. 2, 7 et 20, p. 62, 65 et 71-73), il convient d'ajouter les nombreux tritons naturels, qui n'ont pas été recensés et qui proviennent tous de sépultures. On en trouvera mention dans Pini 1968: 67, n. 790 et on renverra également à Dawkins 1903-04: 197, 202. On notera que la tombe du sarcophage d'Aghia Triada a livré aussi des fragments de triton naturel (Long 1974: 13).

52 Sp. Marinatos et H. Hirmer, Kreta, Thera und des mykenische Hellas, Munich 1973, pl. 10, en haut (l'auteur interprète curieusement le personnage comme une figure masculine: p. 111).

53 Buchholz et Karageorghis, op. cit., no. 1193. Sur l'interprétation de ces rhytons, voir F. Muthmann, Mutter und Quelle. Studien zur Quellenverehrung im Altertum und im Mittelalter, Bâle 1975, p. 198 et 270. 


\section{Summary}

Burial customs manifest during the Aegean Bronze Age close relations with the idea of fertility. This is especially true for the Mycenaean world and it is a further indication of the predilection of the Mycenaeans for the various forms of symbolism. The relation may be expressed by the image of different living beings, more or less directly connected with fertility, or by placing more significant elements such as ostrich eggs within the tomb. The best examples of those elements, however, are the animal head rhyta. Their funeral use is limited to continental late Bronze Age Greece. Their meaning seems to be that of a substitute of a sacrifice pars pro toto which is supposed to supply the deceased with the guarantee of a symbolic and eternal regenerating blood libation in the after-life.

\section{Résumé}

Les coutumes funéraires du domaine égéen présentent au cours de l'âge du Bronze (IIIe et IIe millénaires) des implications nombreuses avec le concept de fécondité. C'est spécialement le cas du monde mycénien, qui donne ici un indice supplémentaire de sa prédilection pour les diverses formes du symbolisme funéraire. La relation peut aller de la simple figuration d'élements qui sont en rapport plus ou moins direct avec la notion de fécondité, jusqu'à l'intégration aux mobiliers funéraires d'objets plus directement évocateurs, comme les oeufs d'autruche. Mais la manifestation la plus révélatrice est donnée par les rhytons en tête animale, dont la destination funéraire est propre à la Grèce continentale du Bronze récent et dont la signification semble être celle d'un sacrifice pars pro toto de substitution qui apporte symboliquement au défunt la garantie d'une libation de sang régénératrice. 
Bibliographie

ANDRONIKOS, M.

1968 - Archaeologia Homerica W, Totenkult, Göttingen.

BAURAIN - DARCQUE

1983 - Un triton en pierre à Malia, $B C H, 107$, pp. 3-73.

CASSIMATIS, $\mathrm{H}$.

1973 - Les rites funéraires à Chypre, $R D A C$, pp. 116-166.

CAUBET, A.

1983 - Les oeufs d'autruche au Proche Orient ancien, $R D A C$, pp. 193198.

DAWKINS, R.M.

1903-04 - Excavations at Palaikastro. III, BSA, 10, pp. 195-235.

DEONNA. W

$19 \overline{9} 22$ - L'oeuf, les dauphins êt ia naissance d'Aphrodite,

Revue de l'histoire des religions, 85, pp. 157-166.

DOUMAS, Chr.

1983 - Thera, Pompeii of the ancient Aegean, Londres.

FINET, A.

1982 - L'oeuf d'autruche, Studia Paulo Naster oblata, II, Orientalia antiqua, Louvain, pp. 68-77.

FOSTER, K.P.

1979 - Aegean Faience of the Bronze Age, New Haven et Londres.

GESELL, G.C.

1985 - Town, Palace and House Cult in Minoan Crete, Göteborg.

HOOD, S.

1978 - The Arts in Prehistoric Greece, Harmondsworth.

KARO, G.

1930-33 - Die Schachtgräber von Mykenai, Munich.

KOEHL, R.B.

1981 - The Function of Aegean Bronze Age Rhyta, Sanctuaries and Cults in the Aegean Bronze Age, Proceedings of the First International Symposium at the Swedish Institute in Athens (1980), Stockholm, pp. 179-188.

KORRES, G. St.

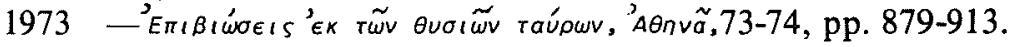

KORRES, G. St.

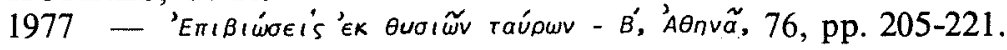

KUBBLR, K.

1954 - Kerameikos VI. Die Nekropole des 10. bis 8. Jahrhunderts, Berlin. 


\section{LAFFINEUR, R.}

1985 - Iconographie minoenne et iconographie mycénienne à l'époque des tombes à fosse, L'iconographie minoenne. Actes de la Table Ronde d'Athènes (1983), BCH, suppl. VI, pp. 245-266.

LONG, Ch.R.

1974 - The Ayia Triadha Sarcophagus. A Study of Late Minoan and Mycenaean Funerary Practices and Beliefs, Göteborg.

MARINATOS, $\mathrm{Sp}$.

1967 - Problemi archeologici e filologici di Pilo, $S M E A, 3$, pp. 6-25.

PARIBENI, R.

1908 - Il sarcofago dipinto di Haghia Triada, Mon. Ant., 19, pp. 20-74.

PETIT, Fr.

1984 - Les rhytons égéens en forme de tête animale, Art \& Fact. Revue des historiens d'art, des archéologues et des orientalistes de l'Université de Liège, 3, pp. 71-80.

PINI, I.

1968 - Beiträge zur minoischen Gräberkunde, Weisbaden.

PLATON, N.

1971 - Zakros. The Discovery of a Lost Place of Ancient Crete, New York.

RENFREW, C.

1981a - Questions of Minoan and Mycenaean Cult, Sanctuaries and Cults in the Aegean Bronze Age, Proceedings of the First International Symposium at the Swedish Institute in Athens (1980), Stockholm, pp. 27-33.

$1981 \mathrm{~b}$ - The Sanctuary at Phylakopi, Sanctuaries and Cults..., pp. 67-80.

SAKELLARAKIS, J.A.

1970 - Das Kuppelgrab A von Archanes und das kretisch-mykenische Tieropferritual, $P Z, 45$, pp. 135-219.

SOMVILLE, $P$.

1984 - Le dauphin dans la religion grecque, Revue de l'histoire des religions, 201, pp. 3-24.

TOUCHAIS, G.

1978 - Chronique des fouilles et découvertes archéologiques en Grèce en $1977, B C H, 102$, pp. 641-770.

TUCHELT, K1.

1962 - Tiergefässe in Kopf - und Protomengestalt, Berlin.

VERMEULE, E.

1975 - The Art of the Shaft Graves of Mycenae, Cincinnati.

XANTHOUDIDES, St.

1924 - The Vaulted Tombs of Mesara, Londres. 